\title{
Clinically Significant Prostate Cancer Detection with Various Prostate Sampling Schemes Based on Different csPCa Definitions
}

\section{Fei Wang}

Second Affiliated Hospital of Soochow University

\section{Tong Chen}

Second Affiliated Hospital of Soochow University

\section{Meng Wang}

Second Affiliated Hospital of Soochow University

\section{Hanbing Chen}

Second Affiliated Hospital of Soochow University

\section{Caishan Wang}

Second Affiliated Hospital of Soochow University

\section{Peiqing Liu}

Second Affiliated Hospital of Soochow University

\section{Songtao Liu}

Second Affiliated Hospital of Soochow University

Jing Luo

Second Affiliated Hospital of Soochow University

Qi Ma ( $D$ maqisz@126.com )

Second Affiliated Hospital of Soochow University

\section{Lijun Xu}

Second Affiliated Hospital of Soochow University

\section{Research Article}

Keywords: clinically significant prostate cancer, contralateral, ipsilateral, systematic biopsy, targeted biopsy

Posted Date: October 11th, 2021

DOl: https://doi.org/10.21203/rs.3.rs-911714/v1

License: (c) (1) This work is licensed under a Creative Commons Attribution 4.0 International License. Read Full License 
Version of Record: A version of this preprint was published at BMC Urology on December 1st, 2021. See the published version at https://doi.org/10.1186/s12894-021-00949-7. 


\section{Abstract}

Background Combining targeted biopsy (TB) with systematic biopsy (SB) is currently recommended as the first-line biopsy method by the European Association of Urology (EAU) guidelines in patients diagnosed with prostate cancer ( $\mathrm{PCa}$ ) with an abnormal magnetic resonance imaging (MRI). The combined SB and TB indeed detected an additional number of patients with clinically significant prostate cancer (csPCa); however, it did so at the expense of a concomitant increase in biopsy cores. Our study aimed to evaluate if ipsilateral SB (ipsi-SB) + TB or contralateral SB (contra-SB) + TB could achieve almost equal csPCa detection rates as SB + TB using fewer cores based on a different csPCa definition.

Methods Patients with at least one positive prostate lesion were prospectively diagnosed by MRI. The combination of TB and SB was conducted in all patients. We compared the csPCa detection rates of the following four hypothetical biopsy sampling schemes with those of $S B+T B$ : SB, TB, ipsi-SB + TB, and contra-SB + TB.

Results The study enrolled 279 men. The median core of SB, TB, ipsi-SB + TB, and contra-SB + TB was 10, 2, 7 and 7, respectively; they all differed significantly from SB + TB $(P<0.001)$. ipsi-SB + TB detected significantly more patients with csPCa than contra-SB + TB based on the EAU guidelines $(P=0.042)$. ipsi$\mathrm{SB}+\mathrm{TB}$ and contra-SB + TB detected almost equal number of csPCa on the basis of the Epstein criteria $(P=1.000)$. Compared with $S B+T B$, each remaining method detected significantly fewer patients with csPCa regardless of the definition $(P<0.001)$ except ipsi-SB $+T B$, which achieved an almost equivalent csPCa detection rate to that of SB + TB on the grounds of D1 $(P=0.066)$.

Conclusions ipsi-SB + TB could detect significantly more patients with csPCa than contra-SB + TB and acquire an almost equivalent cSPCa detection rate to that of $\mathrm{SB}+\mathrm{TB}$ using significantly fewer cores when csPCa was defined as International Society for Urological Pathology (ISUP) 2 or higher.

\section{Background}

The number of patients with prostate cancer ( $\mathrm{PCa}$ ) has increased in the last decades in the USA. An estimated 174,650 and 191,930 men were diagnosed with PCa in 2019 and 2020, respectively, and the number of related deaths was 31,620 and 33,330 , respectively $[1,2]$. PCa is the only tumor diagnosed by blindly puncturing the entire organ rather than just the identified lesion by imaging due to the considerable overlap between benign and malignant lesion appearances in the imaging [3]. Despite its relatively low sensitivities (39\%-75\%) [4] and specificities (40\%-82\%) [5], routine transrectal ultrasound (TRUS)-guided systematic biopsy (SB) remains the diagnostic standard for PCa [6].

The ability to precisely detect PCa using magnetic resonance imaging (MRI) has led to the development of software-assisted MRI-ultrasound fusion guided targeted biopsy (TB). The European Association of Urology (EAU) guidelines currently recommend the combination of TB and SB as the first-line biopsy method for patients with suspected PCa with an abnormal MRI [7]. SB + TB indeed captures an additional number of clinically significant prostate cancer (csPCa), but it does so at the expense of a concomitant 
increase in biopsy cores [8]. The more the biopsy cores, the higher the complication rates, such as hematuria and urinary retention [9]. Thus, exploring a new biopsy method that could achieve an acceptable csPCa detection rate with fewer cores is important.

A previous study has demonstrated that ipsilateral SB (ipsi-SB) + TB could detect more patients with csPCa than contralateral SB (contra-SB) + TB [10]. However, the study was performed on a single definition of csPCa. At present, no universally accepted definition of csPCa exists [11]. Therefore, we performed this study to evaluate the csPCa detection rate of various prostate sampling schemes and verify whether ipsi-SB + TB or contra-SB + TB could achieve almost equal csPCa detection rates to SB + TB using fewer cores based on different csPCa definitions.

\section{Methods}

\section{Patient Selection}

Men with increased serum prostate-specific antigen (PSA) levels (PSA $>4 \mathrm{ng} / \mathrm{mL}$ ) or an abnormal digital rectal examination (DRE) underwent 3.0-T prostate MRI. We included patients whose MRI was positive [with at least one lesion with a Prostate Imaging Reporting and Data System (PI-RADS) score of 3 or greater]. Patients with clinical stage $\mathrm{T}>3$ or metastases, prior treatment for $\mathrm{PCa}$, and under active surveillance were excluded from the study (Fig. 1).

\section{Multiparametric MRI}

Multiparametric magnetic was performed using a 3.0-T scanner with a 32-channel surface coil (Ingenia, Philips, Netherlands). In a nutshell, the study involved triplanar T2-weighted imaging, diffusion-weighted imaging with $a b$ value of $0-100-1000-2000 \mathrm{~s} / \mathrm{mm} 2$, apparent diffusion coefficient maps (calculated by the $b$ value of $100-1000 \mathrm{~s} / \mathrm{mm} 2$ automatically), and dynamic contrast (gadolinium, $2.5 \mathrm{~mL} / \mathrm{s}, 0.1$ $\mathrm{mmol} / \mathrm{kg}$ )-enhanced imaging sequences according to the minimum standards set by consensus guidelines [10]. One genitourinary radiologist interpreted all the lesions visible in MRI according to the PIRADS version 2 on a scale from 1 (no suspicion) to 5 (high suspicion).

\section{Biopsy Procedure}

A fluoroquinolone antibiotic was prescribed 3 days before biopsy to prevent postoperative infection, and an enema was generally performed. A MyLab Twice ultrasound system was used with an EC 1237.5 $\mathrm{MHz}$ transrectal end-fire probe (EsaoteSpA, Genova, Italy) accompanied by an automatic biopsy gun with an $18-G$ needle for sampling.

\section{TB Procedure}


MRI-TRUS registration (i.e., matching of the previously obtained suspicious MRI lesions with the real-time image of the prostate during TRUS biopsy) was performed by software-assisted rigid registration Virtual Navigator (Esaote, Genoa, Italy). Each MRI suspicious lesion was biopsied with at least two cores.

\section{SB Procedure}

The MRI overlay TB was then removed, and a second physician performed an SB with ultrasonographic guidance alone. The standard 10-core biopsy was obtained from the lateral and medial aspects of the base and midgland, and the apical prostate of the left and right sides (Fig. 2).

\section{Hypothetical Biopsy Sampling Schemes and Different Definitions of csPCa}

We hypothesized four biopsy sampling schemes in this study: SB only, TB only, ipsi-SB + TB, and contraSB + TB. Among these, SB + TB was regarded as the reference. csPCa was defined according to the EAU guidelines [International Society for Urological Pathology (ISUP) 2 or higher, definition 1 (D1)] [13] or the Epstein criteria [Gleason score (GS) $>6$ or GS 6 with $\geq 50 \%$ of cancer per core involvement or $>2$ cores with cancer, definition 2 (D2)] [14].

\section{Outcomes of Interest}

A comparison of the csPCa detection rate of the four hypothetical biopsy sampling schemes based on different csPCa definitions was our primary endpoint. The secondary endpoint was to assess the diagnostic concordance and upgrading between the aforementioned sampling schemes and SB + TB.

\section{Statistical Analysis}

Data were prospectively collected according to the Standards of Reporting for MRI-targeted Biopsy Studies database [15]. Descriptive statistics were used to describe the patient characteristics. The difference in the needed cores of different biopsy methods was compared by employing the Wilcoxon signed-rank test. We compared the csPCa detection rate of different biopsy strategies head-to-head using the McNemar test. A Cochran's Q test was used for comparing the pathological concordance and upgrading between different biopsy methods. We evaluated the potential predictors of biopsy result upgrading using multivariable logistic regression. All statistical analyses were conducted using SPSS, version 22.0, and a statistical significance level of $5 \%$ was used.

\section{Results}




\section{Baseline Characteristics of Patients}

In this prospective single-center diagnostic study, 279 subjects with a median age of 71 years

[interquartile range (IQR): $62-80$ ] and median PSA of $10.04 \mathrm{ng} / \mathrm{mL}$ (IQR: 6.38-18.00) were enrolled from January 2017 to December 2020 irrespective of the biopsy history. Abnormal DRE was found in 74 men (26.52\%). The patients' demographics are given in Table 1.

Table 1

Patients' baseline characteristics, TRUS findings and MRI findings.

Men, no.

Age, yr (IQR)

$\mathrm{PSA}, \mathrm{ng} / \mathrm{mL}(\mathrm{IQR})$

Suspicious DRE findings, $\mathbf{n}(\%)$

TRUS prostate volume, $\mathrm{mL}$ (IQR)

Men with prior biopsy, $\mathrm{n}(\%)$

Men without biopsy history, $\mathrm{n}(\%)$

Abnormal TRUS findings, $n$ (\%)

Urologists' biopsy experience, yr (IQR)

MRI suspicious lesions per patient, no. (IQR)

Total lesions, no.

PIRADS v2 score, $n$ (\%)

3

4

5

Location

Peripheral zone, $\mathbf{n}(\%)$

Transitional zone, $\mathbf{n}(\%)$
279

$71(65-77)$

$10.04(6.38-18.00)$

74 (26.52)

$57.00(41.00-82.30)$

$89(31.90)$

$190(68.10)$

139 (49.82)

$4(4-5)$

$1(1-1)$

353

$113(32.01)$

$169(47.88)$

$71(20.11)$

$232(65.72)$

$121(34.28)$

Values are presented as median (interquartile range [IQR]). Statistically significant at $P<0.05$. TRUS $=$ transrectal ultrasound; MRI = magnetic resonance imaging; PSA = prostate-specific antigen; DRE = digital rectal examination; PIRADS = Prostate Imaging Reporting and Data System.

\section{Biopsy Cores}


The median core of SB, TB, ipsi-SB + TB, and contra-SB + TB was 10, 2, 7, and 7, respectively; they all differed significantly from SB + TB $(12, P<0.001)$ (Table 2). Obviously, TB showed the best detection of CSPCa for the total number of cores regardless of the definition $(P<0.001)$ and SB performed the worst $(P<0.001)$. A comparison of the csPCa positive core rates of ipsi-SB + TB and contra-SB + TB revealed that the former performed better irrespective of the definition of csPCa $(P<0.001)(T a b l e 2)$.

Table 2

Summary of biopsy cores.

\begin{tabular}{|c|c|c|c|c|c|}
\hline & SB & TB & ipsi-SB+TB & $\begin{array}{l}\text { contra- } \\
\text { SB+TB }\end{array}$ & $S B+T B$ \\
\hline Biopsy cores, no. & $10(10-10)$ & $2(2-2)$ & $7(7-7)$ & $7(7-7)$ & $12(12-12)$ \\
\hline Positive biopsy cores, no. & $1(0-4)$ & $0(0-2)$ & $2(0-5)$ & $2(0-3)$ & $2(0-6)$ \\
\hline $\begin{array}{l}\text { D1 positive core rate, } n \\
(\%)\end{array}$ & $\begin{array}{l}373 \\
(10.69)\end{array}$ & $\begin{array}{l}230 \\
(29.72)\end{array}$ & $\begin{array}{l}509 \\
(20.21)\end{array}$ & $324(12.86)$ & $603(14.14)$ \\
\hline $\mathbf{P}$ & $<0.001$ & $<0.001$ & $<0.001$ & 0.017 & - \\
\hline $\begin{array}{l}\text { D2 positive core rate, } n \\
\text { (\%) }\end{array}$ & $\begin{array}{l}678 \\
(19.43)\end{array}$ & $\begin{array}{l}357 \\
(46.12)\end{array}$ & $\begin{array}{l}817 \\
(32.43)\end{array}$ & $575(22.83)$ & $\begin{array}{l}1035 \\
(24.27)\end{array}$ \\
\hline $\mathbf{P}$ & $<0.001$ & $<0.001$ & $<0.001$ & 0.051 & - \\
\hline
\end{tabular}

\section{csPCa Detection Rates}

In the whole cohort, 104,146 csPCa and 90,146 csPCa were detected by ipsi-SB + TB and contra-SB + TB, respectively, on the basis of $D 1(P=0.042)$ and $D 2(P=1.000)$. SB could detect more patients with csPCa than TB when used alone; however, the difference was insignificant on the grounds of D1 (D1: 82 vs. 80, $P=0.302 ; D 2: 143$ vs. $118, P=0.002)$. Compared with $S B+T B$, each remaining method detected significantly fewer patients with csPCa regardless of the definition of csPCa $(P<0.001)$ except ipsi$\mathrm{SB}+\mathrm{TB}$, which achieved almost the same csPCa detection rate as that of $\mathrm{SB}+\mathrm{TB}$ based on $\mathrm{D} 1(\mathrm{P}=$ 0.066) (Table 3). 
Table 3

Detection rates of csPCa.

\begin{tabular}{|llllll|}
\hline & SB & TB & $\begin{array}{l}\text { ipsi- } \\
\text { SB+TB }\end{array}$ & $\begin{array}{l}\text { contra- } \\
\text { SB+TB }\end{array}$ & SB+TB \\
\hline $\begin{array}{l}\text { Detected D1 csPCa cases, } \mathbf{n} \\
(\%)\end{array}$ & $82(29.39)$ & $80(28.67)$ & $\begin{array}{l}104 \\
(37.28)\end{array}$ & $90(32.26)$ & 106 \\
\hline $\mathbf{P}$ & $<0.001$ & $<0.001$ & 0.066 & $<0.001$ & - \\
\hline $\begin{array}{l}\text { Detected D2 csPCa cases, } \mathbf{n} \\
\text { (\%) }\end{array}$ & 143 & 118 & 146 & $146(52.33)$ & 161 \\
\hline $\mathbf{P}$ & $(51.25)$ & $(42.29)$ & $(52.33)$ & & $(57.71)$ \\
\hline $\begin{array}{l}\text { Statistically significant at } P<0.05 . \text { csPCa=clinically significant prostate cancer; SB=systematic biopsy; } \\
\begin{array}{l}\text { TB=targeted biopsy; ipsi-SB=ipsilateral SB; contra-SB=contralateral SB; D1 = definition 1 (EAU } \\
\text { guidelines); D2= definition 2 (Epstein criteria); GS= Gleason score. }\end{array}\end{array}$ \\
\hline
\end{tabular}

\section{GS Distribution, Concordance, and Upgrading}

The distribution of the GS on each biopsy method could be seen in Fig. 3. It is worth noting that the number of PCa with a GS of 6 detected by SB was more than that by TB $(P<0.001)$, but the number of $P C a$ with a $G S$ of $\geq 7$ detected by both of them was almost equal $(P=0.311)$. ipsi-SB + TB identified the same number of $\mathrm{PCa}$ as that of contra-SB $+\mathrm{TB}(\mathrm{P}=1.000)$ but higher number of patients with a $\mathrm{GS}$ of $\geq 7(P<0.001)$ and fewer patients with a GS of $6(P<0.001)$.

ipsi-SB detected 92 patients with a higher GS, and 38 patients were still detected after combining with TB. Of the 38 patients, 9 had a GS of 6,14 had a GS of $\leq 6$ on contra-SB + TB to $\geq 3+4$ on ipsi-SB + TB, and the remaining 15 were concordant patients (Fig. 4a). The upgrading of a patient from a GS of $\leq 6$ in one biopsy method to higher than a GS of $\leq 6$ in another was considered as insignificantly upgraded. A patient upgrading from a GS of $\leq 6$ in one biopsy method to a GS of $\geq 3+4$ in another was considered as significantly upgraded. A patient upgrading from a GS of $\geq 3+4$ in one biopsy method to higher than a GS of $\geq 3+4$ in another was considered concordant. Details of the 38 upgrading patients on ipsi-SB are summarized in Supplement Table 1.

In contrast, 17 subjects were identified with a higher GS on contra-SB compared with ipsi-SB, and only 10 additional upgrades occurred after combining with TB. Among them, eight patients had a GS of 6 , one had a GS of 6 on ipsi-SB + TB to a GS of 8 on contra-SB + TB, and one had a GS of 9 on ipsi-SB + TB to a GS of 10 on contra-SB + TB (Fig. 4b). Details of the 10 upgrading patients on contra-SB are summarized in Supplement Table 2.

\section{Potential Predictors of GS Upgrading on ipsi-SB + TB and contra-SB + TB}


We evaluated the potential predictors of GS upgrading on ipsi-SB + TB and contra-SB + TB. For all 38 patients with a higher GS on ipsi-SB + TB, decreased TRUS prostate volume, prior biopsy history, lesion located in the peripheral zone (PZ), and higher PSA level were associated with GS upgrading. Among them, prior biopsy history had the strongest association with GS upgrading [odds ratio (OR): $2.365 ; \mathrm{P}=$ 0.008] (Table 4). In the 14 significantly upgrading patients on ipsi-SB + TB, decreased TRUS prostate volume and lesion located in the PZ remained significant (Table 4).

Table 4

Associated predictors of Gleason score upgrading on ipsi-SB+TB.

\begin{tabular}{|c|c|c|c|c|c|c|}
\hline & \multicolumn{3}{|c|}{$\begin{array}{l}38 \\
\text { upgrading }\end{array}$} & \multicolumn{3}{|c|}{$\begin{array}{l}14 \text { significantly } \\
\text { upgrading }\end{array}$} \\
\hline & OR & $95 \% \mathrm{Cl}$ & $\begin{array}{l}P \\
\text { value }\end{array}$ & OR & $95 \% \mathrm{Cl}$ & $\begin{array}{l}\mathrm{P} \\
\text { value }\end{array}$ \\
\hline $\begin{array}{l}\text { TRUS prostate } \\
\text { volume } \\
\text { (per } 10 \text { volume) }\end{array}$ & 0.970 & $\begin{array}{l}0.950- \\
0.990\end{array}$ & 0.004 & 0.980 & $0.950-1.012$ & 0.022 \\
\hline $\begin{array}{l}\text { Biopsy history } \\
\text { (yes or no) }\end{array}$ & 2.365 & $\begin{array}{l}0.903- \\
6.192\end{array}$ & 0.008 & - & - & - \\
\hline $\begin{array}{l}\text { Location } \\
\text { (PZ or TZ) }\end{array}$ & 1.949 & $\begin{array}{l}0.713- \\
5.324\end{array}$ & 0.019 & 8.424 & $1.201-59.065$ & 0.032 \\
\hline $\begin{array}{l}\text { PSA } \\
\text { (per ng/mL) }\end{array}$ & 1.001 & $\begin{array}{l}0.999- \\
1.004\end{array}$ & 0.028 & - & - & - \\
\hline
\end{tabular}

Prior biopsy history (OR: 3.148; $\mathrm{P}=0.021$ ) and inadequate biopsy experience (OR: $0.701 ; \mathrm{P}=0.032$ ) were associated with a GS of 10 upgrading on contra-SB + TB (Table 5).The basic characteristics of the only patient with significant upgrading on contra-SB + TB were as follows: age 82 years, PSA $30.24 \mathrm{ng} / \mathrm{mL}$, DRE (+), TRUS prostate volume $66.9 \mathrm{~mL}$, prior biopsy history, abnormal TRUS findings, urologists' biopsy experience 5, MRI suspicious lesions 1, maximum PI-RADS 5, and lesion position PZ. 
Table 5

Associated predictors of 10 Gleason score upgrading on contra-SB+TB.

\begin{tabular}{|lccc|}
\hline & OR & $95 \% \mathrm{Cl}$ & Pvalue \\
\hline $\begin{array}{l}\text { Biopsy history } \\
\text { (yes or no) }\end{array}$ & 3.148 & $0.527-18.802$ & 0.021 \\
\hline $\begin{array}{l}\text { Urologists' biopsy experience } \\
\text { (per yr) }\end{array}$ & 0.701 & $0.349-1.409$ & 0.032 \\
\hline $\begin{array}{l}\text { Statistically significant at } P<0.05 . \text { SB=systematic biopsy; TB=targeted biopsy; contra-SB=contralateral } \\
\text { SB; OR = odds ratio; } \mathrm{Cl}=\text { confidence interval. }\end{array}$ \\
\hline
\end{tabular}

\section{Discussion}

An SB is a relatively cost-effective and nonoperator-dependent method of detecting PCa and does not need specialized equipment. However, the method suffers from relatively lower diagnostic accuracy and more biopsy cores. Despite several limitations, systematic sampling of the prostate with different core numbers (commonly 10-12 cores) still represents an integral aspect of diagnosing or excluding PCa [16]. The MRI pathway (MRI with or without TB) has been increasingly used for the detection and risk stratification of csPCa $[17,18]$. A systematic review of the published studies showed that MRI-targeted biopsies resulted in detecting more patients with csPCa and fewer patients with indolent PCa using fewer cores compared with SB [19].

In this study, TB indeed had a significantly higher csPCa positive core rate than SB; however, SB still could detect significantly more patients with csPCa than TB at least based on D2. Similar results were reported by Hakozaki et al. [20] with a higher csPCa detection rate in the TB group; they defined csPCa according to D2 in this context. More patients with csPCa were also detected by SB than by TB on the grounds of D1, although the difference was insignificant in this article. This finding was compatible with that of a previous similar study (Radtke et al. [21]), which reported that the csPCa (in line with D1 in our study) detection rate was higher using SB alone than using TB alone. Compared with D1, D2 of csPCa is more inclusive. For example, cancer with a GS of 6 and three cores would be considered clinically insignificant prostate cancer (icsPCa) on the grounds of D1 but csPCa based on D2. In the series by Filson et al. [22], TB offered the potential to identify more higher-risk patients with $\mathrm{PCa}$. As a consequence, although SB still detected more patients with csPCa than TB, the difference was insignificant when using the stricter D1 of csPCa. Therefore, TB was nonsuperior to SB in terms of detecting csPCa at least in the current study.

In an early study, Ploussard et al. [23] evaluated the added value of concomitant SB for predicting the final grade group in patients with positive MRI findings who underwent TB. The results showed that SB reclassified a non-negligible proportion of patients in a higher-risk category and modified the final treatment decision-making. As a consequence, SB should not be omitted at least in MRI-positive cases, 
just identical to the EAU guidelines [7]. Although SB + TB indeed led to the detection of more PCa and csPCa among patients with MRI-visible lesions, the cores were also increased [8].

Recently, many different modified sampling schemes have emerged to increase the detection of aggressive tumors and decrease biopsy cores and concomitant complications [24]. Freifeld et al. [10] retrospectively enrolled a cohort of 116 men with a single suspicious lesion on MRI and concluded that $\mathrm{TB}+$ ipsi-SB could detect more patients with csPCa while mitigating the detection of icsPCa compared with $\mathrm{TB}+$ contra-SB. Our findings were consistent with those of Freifeld when we used nearly the same definition of csPCa (D1). ipsi-SB + TB also performed better in terms of a positive core rate than contra$\mathrm{SB}+\mathrm{TB}$ irrespective of the definition of csPCa. However, the two methods had an equivalent detection of csPCa based on D2 in this study. Thus, no matter which csPCa definition was chosen in this study, ipsi$\mathrm{SB}+\mathrm{TB}$ did not perform less than contra-SB + TB in the detection of patients with csPCa.

In this study, both ipsi-SB + TB and contra-SB + TB detected fewer patients with csPCa regardless of the definition of csPCa when compared with $\mathrm{SB}+\mathrm{TB}$. The aforementioned findings were identical with those of Freifeld et al. [10], who found that patients were more or less missed or misclassified by TB alone, TB + ipsi-SB, and TB + contra-SB. However, slightly different from the previous study, ipsi-SB + TB detected an almost equal number of patients with csPCa as SB + TB based on D1 in this study. This provided us a novel biopsy scheme that could acquire an equivalent csPCa detection value to SB + TB using significantly fewer cores.

Further, we analyzed the specific GS of each biopsy method. Also, we found that ipsi-SB + TB identified the same number of PCa as contra-SB+TB did but with a higher number of patients with a GS of $\geq 7$ and fewer patients with a GS of 6 . With respect to the upgrading condition, combining ipsi-SB and contra-SB + TB led to 38 GS upgrading; however, combining contra-SB and ipsi-SB +TB only resulted in $10 \mathrm{GS}$ upgrading. Also, a large number of the 38 patients upgraded from GS $\leq 6$ to GS $\geq 7$; conversely, $80 \%$ of the 10 cases with no cancer on ipsi-SB + TB were diagnosed with PCa with a GS of 6 when combined with contra-SB. Recently, as a result of the widespread use of PSA testing, the incidence of PCa has increased (including icsPCa) [25]. Also, after many years of aggressive treatment of $\mathrm{PCa}$, the reduced overdiagnosis and overtreatment of icsPCa have caught the attention of the urology community [26]. According to the EAU guidelines [27], active surveillance should be discussed for patients at low risk of $\mathrm{PCa}$ (PSA $<10 \mathrm{ng} / \mathrm{mL}$ and GS $<7$ and cT1-2a). Patients at intermediate risk (PSA 10-20 ng/mL or GS 7 or cT2b) and those at high risk of $\mathrm{PCa}$ (PSA $>20 \mathrm{ng} / \mathrm{mL}$ or GS $>7$ or $\mathrm{cT} 2 \mathrm{c}$ ) are strongly recommended to undergo radical prostatectomy. As a result, additional contra-SB results in the overdetection of low-risk $\mathrm{PCa}$, while additional ipsi-SB is more likely to change the patients' recommended treatment strategy.

This study has some limitations. First, the inclusion criteria of this study are confined to patients with abnormal MRI; thus, no statement of cancer missed in the initial MRI could be made. Siddiqui et al. [28] reported that MRI showed a negative predictive value of $98 \%$ for PCa with a GS of 7 or greater; hence, further studies are needed to explore the negative predictive value of MRI. Second, whole-mount histopathology was not the reference specimen when the cancer detection rate was compared. Third, the 
rigid registration system did not allow us to make adjustments; even deformations happened to the prostate by the TRUS probe, although some anatomical landmarks can be used to make cognitive fusion at that time in our study [29]. Finally, the same patient was tested with SB and TB, and biopsy complications, such as hemorrhage and swelling of the first conducted one-TB procedure might have negatively affected the SB.

\section{Conclusions}

ipsi-SB + TB could detect significantly more patients with csPCa than contra-SB + TB and acquire an almost equivalent csPCa detection rate to $\mathrm{SB}+\mathrm{TB}$ using significantly fewer cores when csPCa was defined as ISUP 2 or higher. ipsi-SB + TB identified the same number of PCa with contra-SB + TB in total but a higher number of patients with a GS of $\geq 7$ and fewer patients with a GS of 6 .

\section{Abbreviations}

PCa: prostate cancer

TRUS: transrectal ultrasound

SB: systematic biopsy

MRI: magnetic resonance imaging

TB: targeted biopsy

EAU: European Association of Urology

csPCa: clinically significant prostate cancer

ipsi-SB: ipsilateral SB

contra-SB: contralateral SB

PSA: prostate-specific antigen

DRE: digital rectal examination

PI-RADS: Prostate Imaging Reporting and Data System

ISUP: International Society for Urological Pathology

GS: Gleason score

IQR: interquartile range 
PZ: peripheral zone

OR: odds ratio

icsPCa: insignificant prostate cancer

\section{Declarations}

\section{Ethics approval and consent to participate:}

The study was carried out in accordance with the Declaration of Helsinki and approved by Ethics Committee of the Second Affiliated Hospital of Soochow University. Each participant provided voluntary informed consent before the examinations.

\section{Consent for publication:}

Consent for publication of Fig. 2 was obtained from Ma et al.

\section{Availability of data and materials:}

All data generated or analysed during this study are included in this published article and its supplementary information files.

\section{Competing interests:}

The authors declare that they have no competing interests.

\section{Funding:}

This study was funded by the Natural Science Foundation of Jiangsu Province (grant no. BK20191170) and the Suzhou Municipal Science and Technology Bureau (grant no. SS201855). The funders played no role in design of the study, collection, analysis, and interpretation of data or in writing the manuscript.

\section{Authors' contributions:}

FW and QM conceived and designed the study, participated in the collection of data and data analysis, and drafted the manuscript. LJ X assisted in the design of this research and project development. TC, 
MW, HB C, CS W, PQ L, ST L, JL participated in the collection of data. All authors read and approved the final manuscript.

\section{Acknowledgements:}

Not applicable.

\section{References}

1. Sterling J, Smith K, Farber N, et al. Fourteen-Core Systematic Biopsy That Includes Two Anterior Cores in Men With PI-RADS Lesion $>/=3$ is Comparable With Magnetic Resonance Imagingultrasound Fusion Biopsy in Detecting Clinically Significant Prostate Cancer: A Single-institution Experience. Clin Genitourin Cancer 2020 doi: 10.1016/j.clgc.2020.09.006[published Online First: Epub Date]|.

2. Morris DC, Chan DY, Lye TH, et al. Multiparametric Ultrasound for Targeting Prostate Cancer: Combining ARFI, SWEI, QUS and B-Mode. Ultrasound Med Biol 2020;46(12):3426-39 doi: 10.1016/j.ultrasmedbio.2020.08.022[published Online First: Epub Date]|.

3. Gunzel K, Cash H, Buckendahl J, et al. The addition of a sagittal image fusion improves the prostate cancer detection in a sensor-based MRI /ultrasound fusion guided targeted biopsy. BMC Urol 2017;17(1):7 doi: 10.1186/s12894-016-0196-9[published Online First: Epub Date]|.

4. Postema A, Mischi M, de la Rosette J, Wijkstra H. Multiparametric ultrasound in the detection of prostate cancer: a systematic review. World J Urol 2015;33(11):1651-9 doi: 10.1007/s00345-0151523-6[published Online First: Epub Date]|.

5. Heijmink SW, Futterer JJ, Strum SS, et al. State-of-the-art uroradiologic imaging in the diagnosis of prostate cancer. Acta Oncol 2011;50 Suppl 1:25-38 doi: 10.3109/0284186X.2010.578369[published Online First: Epub Date]|.

6. Pinto PA, Chung PH, Rastinehad AR, et al. Magnetic Resonance Imaging/Ultrasound Fusion Guided Prostate Biopsy Improves Cancer Detection Following Transrectal Ultrasound Biopsy and Correlates With Multiparametric Magnetic Resonance Imaging. Journal of Urology 2011;186(4):1281-85 doi: 10.1016/j.juro.2011.05.078[published Online First: Epub Date]|.

7. Guideline. Secondary Guideline. https://uroweb.org/guidelines/.

8. Ahdoot M, Wilbur AR, Reese SE, et al. MRI-Targeted, Systematic, and Combined Biopsy for Prostate Cancer Diagnosis. N Engl J Med 2020;382(10):917-28 doi: 10.1056/NEJMoa1910038[published Online First: Epub Date]|.

9. Borghesi M, Ahmed H, Nam R, et al. Complications After Systematic, Random, and Image-guided Prostate Biopsy. Eur Urol 2017;71(3):353-65 doi: 10.1016/j.eururo.2016.08.004[published Online First: Epub Date]|. 
10. Freifeld $\mathrm{Y}, \mathrm{Xi} \mathrm{Y}$, Passoni $\mathrm{N}$, et al. Optimal sampling scheme in men with abnormal multiparametric MRI undergoing MRI-TRUS fusion prostate biopsy. Urologic oncology 2019;37(1):57-62 doi: 10.1016/j.urolonc.2018.10.009[published Online First: Epub Date]|.

11. Shin T, Smyth TB, Ukimura O, et al. Diagnostic accuracy of a five-point Likert scoring system for magnetic resonance imaging (MRI) evaluated according to results of MRI/ultrasonography imagefusion targeted biopsy of the prostate. BJU Int 2018;121(1):77-83 doi: 10.1111/bju.13972[published Online First: Epub Date]l.

12. Ma Q, Yang DR, Xue BX, et al. Transrectal real-time tissue elastography targeted biopsy coupled with peak strain index improves the detection of clinically important prostate cancer. Oncology letters 2017;14(1):210-16 doi: 10.3892/ol.2017.6126[published Online First: Epub Date]|.

13. Mottet N, Bellmunt J, Bolla M, et al. EAU-ESTRO-SIOG Guidelines on Prostate Cancer. Part 1: Screening, Diagnosis, and Local Treatment with Curative Intent. Eur Urol 2017;71(4):618-29 doi: 10.1016/j.eururo.2016.08.003[published Online First: Epub Date]|.

14. Epstein JI, Walsh PC, Carmichael M, Brendler CB. Pathologic and clinical findings to predict tumor extent of nonpalpable (stage T1c) prostate cancer. Jama 1994;271(5):368-74

15. Moore CM, Kasivisvanathan V, Eggener S, et al. Standards of reporting for MRI-targeted biopsy studies (START) of the prostate: recommendations from an International Working Group. Eur Urol 2013;64(4):544-52 doi: 10.1016/j.eururo.2013.03.030[published Online First: Epub Date]|.

16. Bjurlin MA, Taneja SS. Standards for prostate biopsy. Curr Opin Urol 2014;24(2):155-61 doi: 10.1097/mou.0000000000000031[published Online First: Epub Date]|.

17. Weinreb JC, Barentsz JO, Choyke PL, et al. PI-RADS Prostate Imaging - Reporting and Data System: 2015, Version 2. Eur Urol 2016;69(1):16-40 doi: 10.1016/j.eururo.2015.08.052[published Online First: Epub Date]|.

18. Turkbey B, Rosenkrantz AB, Haider MA, et al. Prostate Imaging Reporting and Data System Version 2.1: 2019 Update of Prostate Imaging Reporting and Data System Version 2. Eur Urol 2019;76(3):340-51 doi: 10.1016/j.eururo.2019.02.033[published Online First: Epub Date]|.

19. Valerio M, Donaldson I, Emberton M, et al. Detection of Clinically Significant Prostate Cancer Using Magnetic Resonance Imaging-Ultrasound Fusion Targeted Biopsy: A Systematic Review. Eur Urol 2015;68(1):8-19 doi: 10.1016/j.eururo.2014.10.026[published Online First: Epub Date]|.

20. Hakozaki $Y$, Matsushima $\mathrm{H}$, Kumagai J, et al. A prospective study of magnetic resonance imaging and ultrasonography (MRI/US)-fusion targeted biopsy and concurrent systematic transperineal biopsy with the average of 18-cores to detect clinically significant prostate cancer. BMC Urol 2017;17(1):117 doi: 10.1186/s12894-017-0310-7[published Online First: Epub Date]|.

21. Radtke JP, Schwab C, Wolf MB, et al. Multiparametric Magnetic Resonance Imaging (MRI) and MRITransrectal Ultrasound Fusion Biopsy for Index Tumor Detection: Correlation with Radical Prostatectomy Specimen. Eur Urol 2016;70(5):846-53 doi: 10.1016/j.eururo.2015.12.052[published Online First: Epub Date]l. 
22. Filson CP, Natarajan S, Margolis DJ, et al. Prostate cancer detection with magnetic resonanceultrasound fusion biopsy: The role of systematic and targeted biopsies. Cancer 2016;122(6):884-92 doi: 10.1002/cncr.29874[published Online First: Epub Date]|.

23. Ploussard G, Beauval JB, Lesourd M, et al. Added Value of Concomitant Systematic and Fusion Targeted Biopsies for Grade Group Prediction Based on Radical Prostatectomy Final Pathology on Positive Magnetic Resonance Imaging. J Urol 2019;202(6):1182-87 doi:

10.1097/ju.0000000000000418[published Online First: Epub Date]|.

24. Loeb S, Vellekoop A, Ahmed HU, et al. Systematic review of complications of prostate biopsy. Eur Urol 2013;64(6):876-92 doi: 10.1016/j.eururo.2013.05.049[published Online First: Epub Date]|.

25. Borkowetz A, Platzek I, Toma M, et al. Comparison of systematic transrectal biopsy to transperineal magnetic resonance imaging/ultrasound-fusion biopsy for the diagnosis of prostate cancer. BJU Int 2015;116(6):873-9 doi: 10.1111/bju.13023[published Online First: Epub Date]|.

26. Albisinni S, Aoun F, Noel A, et al. Are concurrent systematic cores needed the time of targeted biopsy in patients with prior negative prostate biopsies? Progres en urologie: journal de l'Association francaise d'urologie et de la Societe francaise d'urologie 2018;28(1):18-24 doi:

10.1016/j.purol.2017.10.001[published Online First: Epub Date]|.

27. Mottet N, van den Bergh RCN, Briers E, et al. EAU-EANM-ESTRO-ESUR-SIOG Guidelines on Prostate Cancer-2020 Update. Part 1: Screening, Diagnosis, and Local Treatment with Curative Intent. Eur Urol 2021;79(2):243-62 doi: 10.1016/j.eururo.2020.09.042[published Online First: Epub Date]|.

28. Siddiqui MM, Rais-Bahrami S, Turkbey B, et al. Comparison of MR/ultrasound fusion-guided biopsy with ultrasound-guided biopsy for the diagnosis of prostate cancer. Jama 2015;313(4):390-7 doi: 10.1001/jama.2014.17942[published Online First: Epub Date]|.

29. Venderink W, Bomers JG, Overduin CG, et al. Multiparametric Magnetic Resonance Imaging for the Detection of Clinically Significant Prostate Cancer: What Urologists Need to Know. Part 3: Targeted Biopsy. Eur Urol 2020;77(4):481-90 doi: 10.1016/j.eururo.2019.10.009[published Online First: Epub Date]|.

\section{Figures}




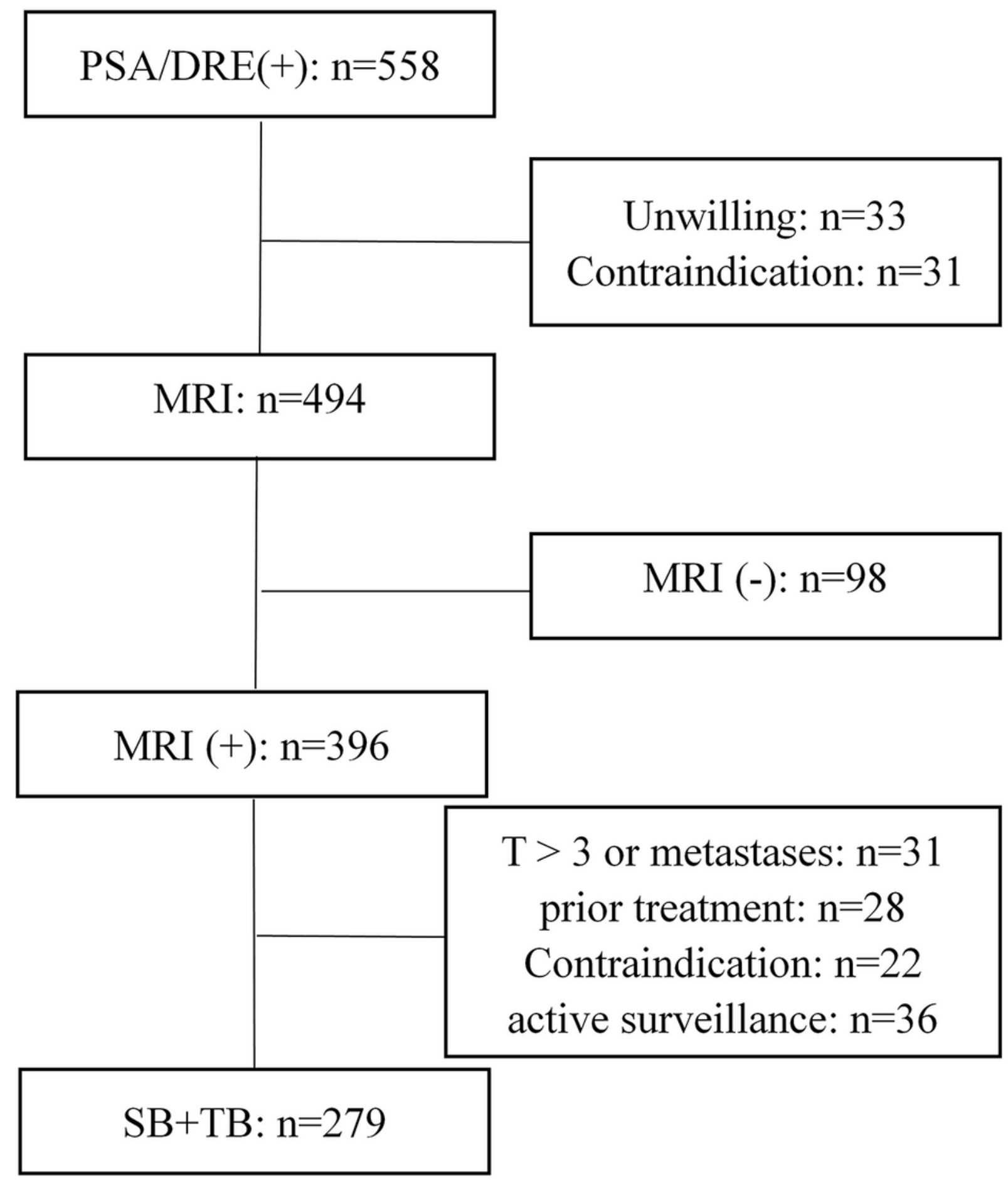

Figure 1

Flowchart for study inclusion/exclusion. PSA = prostate-specific antigen; DRE = digital rectal examination; $\mathrm{MRI}$ = magnetic resonance imaging; $\mathrm{SB}$ = systematic biopsy; TB=targeted biopsy. 


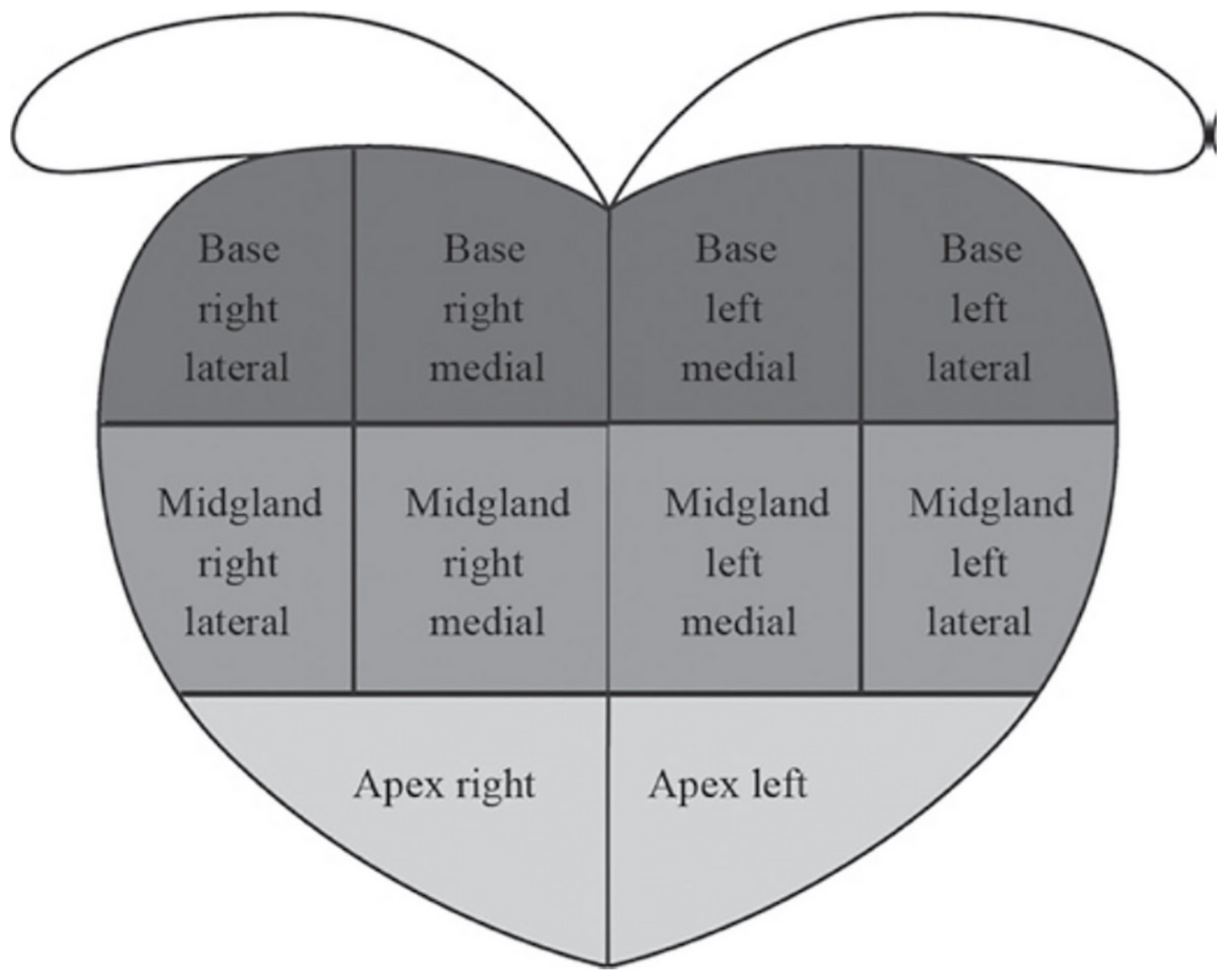

Figure 2

The standard 10-core biopsy. 10 cores obtained from the lateral and medial aspects of the base and midgland and apical prostate of the left and right side. (Reprinted with the kind permission from Ma et al., 2017 [12]). 


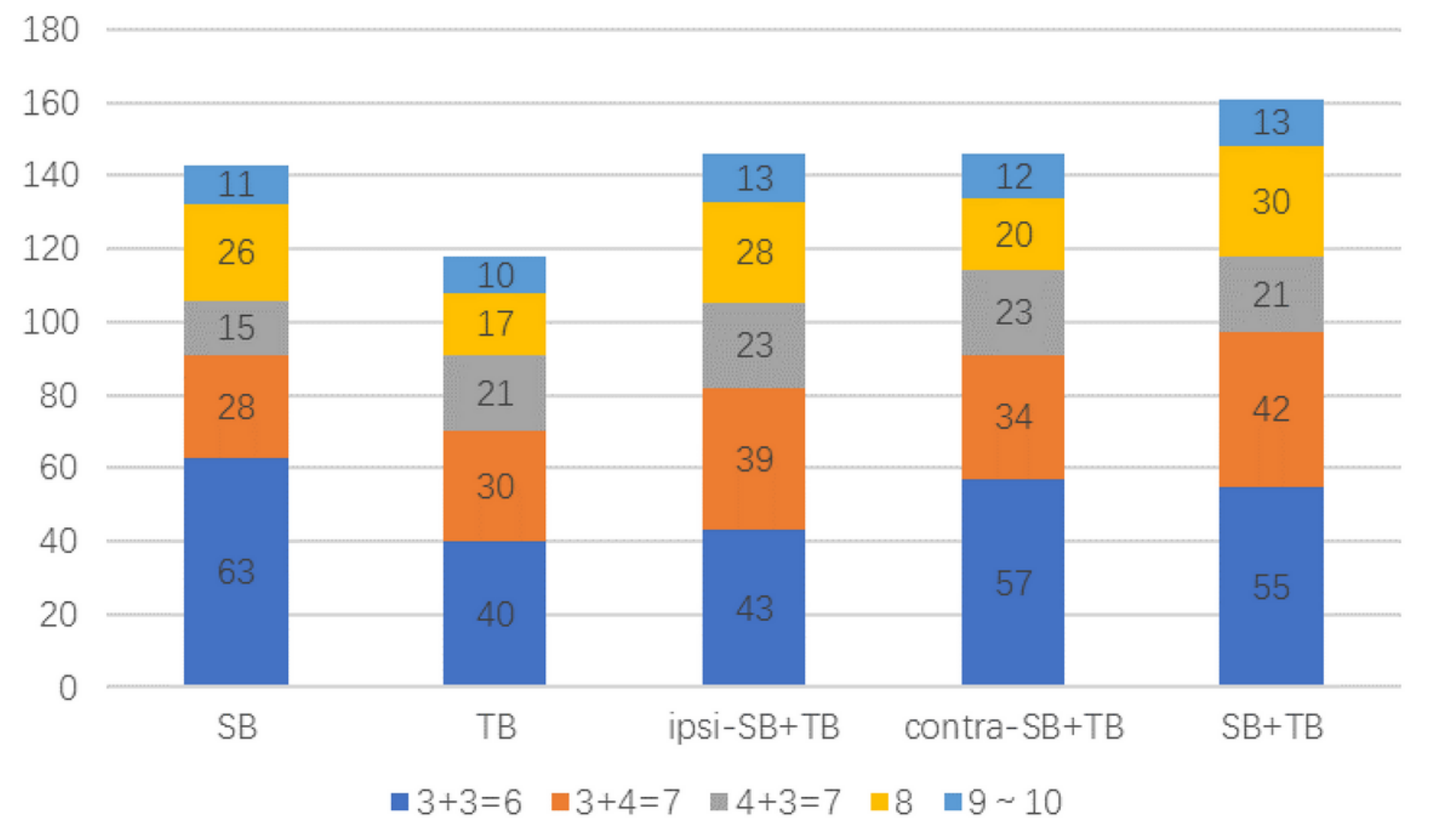

Figure 3

Distribution of Gleason score on each biopsy method.

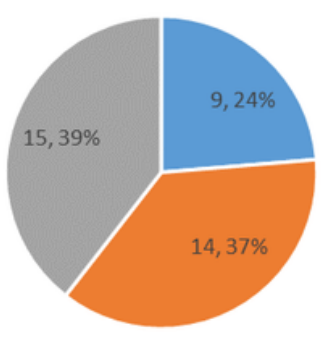

- contra-SB(-), TB(-), ipsi-SB 3+3=6

= contra-SB, $\mathrm{TB}(-) / 3+3=6$, ipsi- $\mathrm{SB} \geq 3+4=7$

- contra-SB, TB, ipsi-SB $\geq 3+4=7$, ipsi-SB>contra-SB b

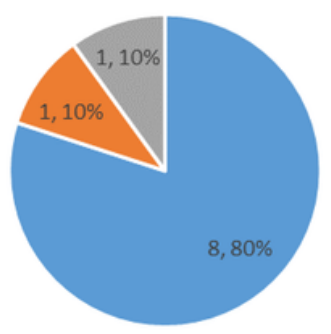

- ipsi-SB(-), TB(-), contra-SB 3+3=6

- ipsi-SB, TB $(-) / 3+3=6$, contra-SB $\geq 3+4=7$

- ipsi-SB, TB, contra-SB $\geq 3+4=7$, contra-SB>ipsi-SB

\section{Figure 4}

Gleason score concordance and upgrading seen (a) on ipsi-SB and (b) on contra-SB.

\section{Supplementary Files}

This is a list of supplementary files associated with this preprint. Click to download. 
- SupplementTable1.docx

- SupplementTable2.docx 